\title{
Localized Delaunay Triangulation with Application in Ad Hoc Wireless Networks
}

\author{
Xiang-Yang Li, Member, IEEE, Gruia Calinescu, Member, IEEE, \\ Peng-Jun Wan, Member, IEEE, and Yu Wang, Student Member, IEEE
}

\begin{abstract}
Several localized routing protocols guarantee the delivery of the packets when the underlying network topology is a planar graph. Typically, relative neighborhood graph (RNG) or Gabriel graph (GG) is used as such planar structure. However, it is well-known that the spanning ratios of these two graphs are not bounded by any constant (even for uniform randomly distributed points). Bose et al. [11] recently developed a localized routing protocol that guarantees that the distance traveled by the packets is within a constant factor of the minimum if Delaunay triangulation of all wireless nodes is used, in addition, to guarantee the delivery of the packets. However, it is expensive to construct the Delaunay triangulation in a distributed manner. Given a set of wireless nodes, we model the network as a unit-disk graph (UDG), in which a link $u v$ exists only if the distance $\|u v\|$ is at most the maximum transmission range. In this paper, we present a novel localized networking protocol that constructs a planar 2.5-spanner of UDG, called the localized Delaunay triangulation (LDEL), as network topology. It contains all edges that are both in the unit-disk graph and the Delaunay triangulation of all nodes. The total communication cost of our networking protocol is $O(n \log n)$ bits, which is within a constant factor of the optimum to construct any structure in a distributed manner. Our experiments show that the delivery rates of some of the existing localized routing protocols are increased when localized Delaunay triangulation is used instead of several previously proposed topologies. Our simulations also show that the traveled distance of the packets is significantly less when the FACE routing algorithm is applied on LDEL, rather than applied on GG.
\end{abstract}

Index Terms-Delaunay triangulation, localized method, planar, routing, spanner, topology control, wireless networks.

\section{INTRODUCTION}

$W^{\mathrm{s}}$ E consider a wireless ad hoc network (or sensor network) with all nodes distributed in a two-dimensional plane. Assume that all wireless nodes have distinctive identities and each static wireless node knows its position information, ${ }^{1}$ either through a low-power Global Position System (GPS) receiver or through some other way. For simplicity, we also assume that all wireless nodes have the same maximum transmission range and we normalize it to one unit. By one-hop broadcasting, each node $u$ can gather the location information of all nodes within the transmission range of $u$. Consequently, all wireless nodes $V$ together, define a unit-disk graph $U D G(V)$, which has an edge $u v$ if and only if the Euclidean distance $\|u v\|$ between $u$ and $v$ is less than one unit. Throughout this paper, a broadcast by a node $u$ means $u$ sends the message to all nodes within its transmission range. In wireless ad hoc networks, the radio signal sent out by a node $u$ can be received by all nodes within the transmission range of $u$. The main communication cost in wireless networks is to send out the signal while the receiving cost of a message is

1. More specifically, it is enough for our protocol when each node knows the relative position of its one-hop neighbors. The relative position of neighbors can be estimated by the direction of arrival and strength of signal.

- The authors are with the Department of Computer Science, Illinois Institute of Technology, Chicago, IL 60616.

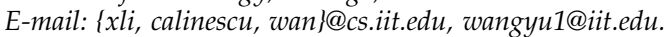

Manuscript received 15 July 2002; revised 12 Nov. 2002; accepted 21 May 2003.

For information on obtaining reprints of this article, please send e-mail to: tpds@computer.org, and reference IEEECS Log Number 116958. neglected here. Consequently, throughout this paper, we are interested in designing a protocol with a small total number of messages sent out by all wireless nodes.

One of the key challenges in the design of ad hoc networks is the development of dynamic routing protocols that can efficiently find routes between two communication nodes. In recent years, a variety of routing protocols [1], [2], [3], [4], [5], [6] targeted specifically for ad hoc environment have been developed. See [7], [8] for a review of most routing protocols.

Several researchers proposed another set of routing protocols, namely, the localized routing, which selects the next node to forward the packets based on the information in the packet header and the position of its local neighbors. Bose and Morin [9] showed that several localized routing protocols guarantee to deliver the packets if the underlying network topology is the Delaunay triangulation of all wireless nodes. They also gave a localized routing protocol based on the Delaunay triangulation such that the total distance traveled by the packet is no more than a small constant factor of the distance between the source and the destination. However, it is expensive to construct the Delaunay triangulation in a distributed manner, and routing based on it might not be possible since the Delaunay triangulation can contain links longer than one unit.

Several researchers also proposed to use some planar network topologies that can be constructed efficiently in a distributed manner. Lin et al. [10] proposed the first localized algorithm that guarantees delivery by memorizing past traffic at nodes. Bose et al. [11] proposed to use the Gabriel graph as the underlying structure for the FACE routing method. Subsequently, Karp et al. [12] discussed, in detail, the medium access layer and conducted experiments 
with moving nodes for the Face routing method. Barriére et al. [13] extended the scheme on graphs which are fuzzy unit graphs; that is, two nodes are connected if their distance is at most $r$, not connected if the distance is at least $R$, and may be connected otherwise. They showed that their algorithm works correctly if $R \leq \sqrt{2} r$. Routing, according to the right-hand rule, which guarantees delivery in planar graphs [9], is also used when simple greedy-based routing heuristics fail.

However, it is well-known [14], [15] that the spanning ratios of both RNG and GG are not bounded by any constant. Here, given a graph $H$, a spanning subgraph $G$ of $H$ is a $t$-spanner if the length of the shortest path connecting any two points in $G$ is no more than $t$ times the length of the shortest path connecting the two points in $H$. Moreover, it was shown by Bose et al. [14] that the spanning ratio of the Gabriel graph on a uniformly random $n$ points set in a square is almost surely at least $\Omega(\sqrt{\log n / \log \log n})$. Thus, no matter how good the routing method is, the spanning ratio achieved by applying the method on the Gabriel graph or on the relative neighborhood graph is at least $\Omega(\sqrt{\log n / \log \log n})$, almost surely. Consequently, to make a localized routing protocol efficient, we need to construct a planar spanner locally. Here, a structure $G$ can be constructed locally if every wireless node $u$ can decide the edges of $G$ incident on $u$, by using only the information of nodes within a constant hops. In this paper, we design a localized algorithm that constructs a planar $t$-spanner for the unit-disk graph, such that some of the localized routing protocols can be applied on it. We obtain a value of approximately 2.5 for the constant $t$. Notice that the spanning ratio achieved by a specific routing method could be much larger than the spanning ratio of the underlying structure. Nonetheless, a structure with a small spanning ratio is necessary for some routing method to possibly perform well.

Our structure is based on the Delaunay triangulation. Given a set of points $V$, the unit Delaunay triangulation, denoted by $U \operatorname{Del}(V)$, is the graph obtained by removing all edges of the Delaunay triangulation $\operatorname{Del}(V)$ that are longer than one unit. It was proven in [16], [17] that $U \operatorname{Del}(V)$ is a $t$-spanner of $U D G(V)$. We then give a localized algorithm that constructs a graph, called localized Delaunay graph $L D e l^{(1)}(V)$. We prove that $\operatorname{LDel}^{(1)}(V)$ is a $t$-spanner by showing that it is a supergraph of $U \operatorname{Del}(V)$. Additionally, we prove that $L \operatorname{Del}^{(1)}(V)$ has thickness two, i.e., it can be decomposed to two planar graphs. We then show how to make the graph $L D e l^{(1)}(V)$ planar efficiently without losing the spanner property. The total communication cost of our approach is $O(n \log n)$ bits, which is optimal within a constant factor. Notice that every node has to send at least one message to its neighbors to notify its existence in any protocol, which implies that the communication cost is at least $n \log n$ bits for any protocol. We assume a node ID can be represented by $\log n$ bits.

The precise worst-case upper bound on the communication costs to construct our planar spanner is $(37 q+13 p+$ 100) $n$ bits, where $q$ is the number of bits required to represent the unique node IDs, and $p$ is the number of bits required to represent the geometric position of a node. Our method operates in an asynchronous environment, and we do not count the time it takes to build the structure. We do not have nontrivial worst-case upper bounds on maintaining the structure due to changes in the network configuration, but believe the average case (assuming changes are random) is acceptable due to the fact that, because the structure is planar and planar graphs have at most $3 n-6$ edges, the average degree of a node in the structure is at most 6 . Our belief is also based on the fact that inserting nodes in a Delaunay triangulation in a random order results in a $O(n \log n)$ expected-time centralized algorithm [23]. When a node moves, the expected number of edges that are affected is at most 6 . Thus, the average cost of updating the structure is small.

The Gabriel graph can be constructed with $(q+p+1) n$ bits and is very easy to maintain when the network changes. Thus, there is a trade off between the better worst-case spanning ratio when applying the greedy routing schemes on our structure and the higher cost of constructing and maintaining it, compared to the Gabriel graph.

Previously, there were some approaches proposed to approximate the Delaunay triangulation locally. Hu [18] used the Delaunay triangulation to configure the wireless network topology such that a planar graph with a bounded node degree is computed. A major step in his method is that each node $u$ computes all Delaunay edges whose length is no more than the transmission range. It used the Voronoi diagram of node $u$ to compute all such Delaunay edges. However, this approach will not always work. A simple observation is that, in order to determine whether an edge $u v$ belongs to the Delaunay triangulation, we have to check whether certain circles passing through $u$ and $v$ are empty (not containing wireless nodes in their interior). Obviously, in the worst case, the circumradius of such a circle could be infinity even when the edge length $u v$ is bounded, implying that we may have to check all nodes. Due to space limit, we omit the detail of why the method in [18] will not work. Moreover, it is unknown whether Hu's structure has a constant spanning ratio.

A related result was published by Gao et al. [17]. The conference version of this paper, [16], was obtained independently and was submitted before [17] was published. Gao et al. [17] proposed another structure, called restricted Delaunay graph RDG and showed that it has good spanning ratio properties and described how to maintain it locally. They called any planar graph containing $U \operatorname{Del}(V)$ a restricted Delaunay graph. They described a distributed algorithm to maintain a RDG such that, at the end of the algorithm, each node $u$ maintains a set of edges $E(u)$ incident to $u$. Those edges $E(u)$ satisfy that 1 ) each edge in $E(u)$ has length at most one unit; 2$)$ the edges are consistent, i.e., an edge $u v \in E(u)$ if and only if $u v \in E(v) ; 3)$ the graph obtained is planar; and 4) $U \operatorname{Del}(V)$ is in the union of all edges $E(u)$.

Their algorithm works as follows: First, each node $u$ acquires the position of its 1-hop neighbors $N_{1}(u)$ and computes the Delaunay triangulation $\operatorname{Del}\left(N_{1}(u)\right)$ on $N_{1}(u)$, including $u$ itself. In the second step, each node $u$ sends $\operatorname{Del}\left(N_{1}(u)\right)$ to all of its neighbors. Let $E(u)=\{u v \mid u v \in$ $\left.\operatorname{Del}\left(N_{1}(u)\right)\right\}$. For each edge $u v \in E(u)$ and for each $w \in N_{1}(u)$, if $u$ and $v$ are in $N_{1}(w)$ and $u v \notin \operatorname{Del}\left(N_{1}(w)\right)$, then node $u$ deletes edge $u v$ from $E(u)$. They proved that, when the above steps are finished, the resulting edges $E(u)$ satisfy the four 
properties listed above. The communication cost could be as large as $\Theta\left(n^{2}\right)$, and the computation cost could be as large as $\Theta\left(n^{3}\right)$, which are much higher than ours.

Recently, Li et al. [19] also proposed another structure partial Delaunay triangulation (PDT), which is a subset of $U \operatorname{Del}(V)$ and a superset of Gabriel graph, for scatternet formation in Bluetooth network. Unfortunately, PDT does not have constant bounded spanning ratio.

Bose et al. [11] and Karp et al. [12] proposed similar algorithms that route the packets using the Gabriel graph to guarantee the delivery. Applying the routing methods proposed in [11], [12] on the planarized localized Delaunay graph $L D e l^{(1)}(V)$, a better performance is expected because the localized Delaunay triangulation is denser compared to the Gabriel graph, but still with $O(n)$ edges. Our simulations show that the delivery rates of several localized routing protocols are increased when the localized Delaunay triangulation is used. In our experiments, several simple local routing heuristics, applied on the localized Delaunay triangulation, have always successfully delivered the packets, while other heuristics were successful in more than 90 percent of the random instances. The greedy-based localized routing schemes may still fail to deliver the packet on localized Delaunay triangulation. Because the constructed topology is planar, we can use the right-hand rule or Face routing to guarantee the delivery of the packets from source node to the destination when simple heuristics fail. The experiments also show that several localized routing algorithms (notably, compass routing [20] and greedy routing) also result in a path whose length is within a small constant factor of the shortest path; we already know such a path exists since the localized Delaunay triangulation is a $t$-spanner. Notice that there is a difference between the spanning ratio of the underlying structure and the spanning ratio achieved by a specific routing algorithm. Obviously, any routing method cannot achieve small spanning ratio when it is applied on a structure with large spanning ratio. We also conduct extensive simulations of the Face routing method [11] and the Greedy Face Routing method (applies the greedy routing whenever possible and uses the Face routing if local minimum occurs) on the localized Delaunay triangulation and the Gabriel graph. For Face routing, we found that the worst-case spanning ratio when localized Delaunay triangulation is used is significantly less than the spanning ratio when Gabriel graph is used, although the average spanning ratio achieved by these two structures are almost the same when the network has no more than 100 nodes. We expect our local Delaunay triangulation to perform better when the number of nodes is significantly large, since the spanning ratio of the Gabriel graph on a set of $n$ random points is $\Omega(\sqrt{\log n / \log \log n})$.

The remainder of the paper is organized as follows: In Section 2, we review some structures that are often used to construct the topology for wireless networks. We define localized Delaunay triangulations $\operatorname{LDel}^{(k)}(V)$ and study their properties in Section 3. Section 4 presents the first localized efficient algorithm that constructs a planar graph, $P L \operatorname{Del}(V)$, which contains $U \operatorname{Del}(V)$ as a subgraph. Thus, $P L D e l(V)$ is a planar $t$-spanner. The correctness of our algorithm is justified in the Appendix. We demonstrate the effectiveness of the localized Delaunay triangulation in Section 5, by studying the performance of various routing protocols on it. We conclude our paper and discuss possible future research directions in Section 6.

\section{Preliminaries}

\subsection{Spanner}

Let $\Pi_{G}(u, v)$ be the shortest path connecting $u$ and $v$ in a weighted graph $G$ and $\left\|\Pi_{G}(u, v)\right\|$ be the length of $\Pi_{G}(u, v)$. Then, a graph $G$ is a $t$-spanner of a graph $H$ if $V(G)=V(H)$ and, for any two nodes $u$ and $v$ of $V(H),\left\|\Pi_{H}(u, v)\right\| \leq$ $\left\|\Pi_{G}(u, v)\right\| \leq t\left\|\Pi_{H}(u, v)\right\|$. With $H$ understood, we also call $t$ the length stretch factor of the spanner $G$.

There are several geometrical structures which are proven to be $t$-spanners for the Euclidean complete graph $K(V)$ of a point set $V$. For example, the Yao graph [21] and the $\theta$-graph [22] have been shown to be $t$-spanners. However, both of these two geometrical structures are not guaranteed to be planar in two dimensions.

Let $\varrho_{G}(u, v)$ be the path found by a unicasting routing method $\varrho$ from node $u$ to $v$ in a weighted graph $G$ and $\left\|\varrho_{G}(u, v)\right\|$ be the length of the path. The spanning ratio achieved by a routing method $\varrho$ is defined as $\max _{G} \max _{u, v}$ $\left\|\varrho_{G}(u, v)\right\| /\|u v\|$.

\subsection{Delaunay Triangulation}

We review the definition of Delaunay triangulation [23]. We assume that all wireless nodes are given as a set $V$ of $n$ nodes in a two-dimensional space. Each node has some computational power. We also assume that there are not four nodes of $V$ that are cocircular. A triangulation of $V$ is a Delaunay triangulation, denoted by $\operatorname{Del}(V)$, if the circumcircle of each of its triangles does not contain any other nodes of $V$ in its interior. A triangle is called the Delaunay triangle if its circumcircle is empty of nodes of $V$ inside. It is wellknown that the Delaunay triangulation $\operatorname{Del}(V)$ is a planar $t$-spanner of the completed Euclidean graph $K(V)$. This was first proven by Dobkin et al. [24] with upper bound $\frac{1+\sqrt{5}}{2} \pi \approx$ 5.08 on $t$. Then, Kevin and Gutwin [25], [22] improved the upper bound on $t$ to be $\frac{2 \pi}{3 \cos _{6}^{\pi}}=\frac{4 \sqrt{3}}{9} \pi \approx 2.42$. The best-known lower bound on $t$ is $\pi / 2$, which is due to Chew [26], and it is widely believed to be the actual upper bound also.

\subsection{Proximity Graphs}

Besides the Delaunay triangulation, various proximity subgraphs of UDG can be defined [27], [28], [29], [30], [21] over a set of $n$ two-dimensional wireless nodes $V$.

For convenience, let $\operatorname{disk}(u, v)$ be the closed disk with diameter $u v$, let $\operatorname{disk}(u, v, w)$ be the circumcircle defined by the triangle $\triangle u v w$, and let $B(u, r)$ be the circle centered at $u$ with radius $r$. Let $x(v)$ and $y(v)$ be the value of the $x$-coordinate and $y$-coordinate of a node $v$, respectively.

The constrained relative neighborhood graph, denoted by $R N G(V)$, consists of all edges $u v$ such that $\|u v\| \leq 1$ and there is no point $w \in S$ such that $\|u w\|<\|u v\|$ and $\|w v\|<\|u v\|$. The constrained Gabriel graph, denoted by $G G(V)$, consists of all edges $u v$ such that $\|u v\| \leq 1$ and the interior of $\operatorname{disk}(u, v)$ does not contain any node from $V$. The constrained $Y$ ao graph with an integer parameter $k \geq 6$, denoted by $\overrightarrow{Y G}_{k}(V)$, is 
defined as follows: At each node $u$, any $k$ equal-separated rays originated at $u$ define $k$ cones. In each cone, choose the closest node $v$ to $u$ with distance at most one, if there is any, and add a directed link $\overrightarrow{u v}$. Ties are broken arbitrarily. Let $Y G_{k}(V)$ be the undirected graph obtained by ignoring the direction of each link in $\overrightarrow{Y G}_{k}(V)$.

The length stretch factor of $R N G(V)$ is at most $n-1$, see [14]; and the length stretch factor of $G G(V)$ is at most $\sqrt{n-1}$, see [31]. Several papers [32], [33], [27] have shown that the Yao graph $Y G_{k}(V)$ has length stretch factor at most $\frac{1}{1-2 \sin \frac{\pi}{\pi}}$. However, the Yao graph is not guaranteed to be planar. The relative neighborhood graph and the Gabriel graph are planar graphs, but they are not spanners for the unit-disk graph. In this paper, we are interested in locally constructing a planar graph that is a spanner of the unitdisk graph.

\subsection{Localized Routing Algorithms}

Let $N_{k}(u)$ be the set of nodes of $V$ that are within $k$ hops of $u$ in the unit-disk graph $U D G(V)$. A node $v \in N_{k}(u)$ is called the $k$-neighbor of the node $u$. Usually, here, the constant $k$ is 1 or 2 , which will be omitted if it is clear from the context. In this paper, we always assume that each node $u$ of $V$ knows its location and identity. Then, after one broadcast by every node, each node $u$ of $V$ knows the location and identity information of all nodes in $N_{1}(u)$. The total communication cost of all nodes to do so is $O(n \log n)$ bits.

A distributed algorithm is a localized algorithm if it uses only the information of all $k$-local nodes of each node plus the information of a constant number of additional nodes. In this paper, we concentrate on the case $k=1$. That is, a node uses only the information of the 1-hop neighbors. A graph $G$ can be constructed locally in the ad hoc wireless environment if each wireless node $u$ can compute the edges of $G$ incident on $u$ by using only the location information of all its $k$-local nodes. In this paper, we design a localized algorithm that constructs a planar $t$-spanner for the unitdisk graph $U D G(V)$ such that some localized routing protocols can be applied on it. The localized construction of the structure is attractive for wireless ad hoc networks due to efficient updating of the structure in mobile environment.

Assume a packet is currently at node $u$, and the destination node is $t$. Several localized routing algorithms that use just the local information of $u$ to route packets (i.e., find the next node $v$ of $u$ ) were developed. Kranakis et al. [20] proposed to use the compass routing, which basically finds the next relay node $v$ such that the angle $\angle v u t$ is the smallest among all neighbors of $u$ in a given topology. Lin et al. [10], Bose et al. [11], and Karp et al. [12] proposed similar greedy routing methods, in which node $u$ forwards the packet to its neighbor $v$ in a given topology which is closest to $t$. Recently, Bose et al. [34], [9], [11] proposed several localized routing algorithms that route a packet from a source node $s$ to a destination node $t$. Specifically, Bose and Morin [9] proposed a localized routing method based on the Delaunay triangulation. They showed that the distance traveled by the packet is within a small constant factor of the distance between $s$ and $t$. They also proved that the compass routing and the greedy routing method guarantee to deliver the packet if the Delaunay triangulation is used.

\section{Local Delaunay Triangulation}

In this section, we define a new topology, called local Delaunay triangulation, which can be constructed in a localized manner. We first introduce some geometric structures and notations to be used in this section. All angles are measured in radians and take values in the range $[0, \pi]$. For any three points $p_{1}, p_{2}$, and $p_{3}$, the angle between the two rays $p_{1} p_{2}$ and $p_{1} p_{3}$ is denoted by $\angle p_{3} p_{1} p_{2}$ or $\angle p_{2} p_{1} p_{3}$. The closed infinite area inside the angle $\angle p_{3} p_{1} p_{2}$, also referred to as a sector, is denoted by $\angle p_{3} p_{1} p_{2}$ without confusion to the angle notation. The triangle determined by $p_{1}, p_{2}$, and $p_{3}$ is denoted by $\triangle p_{1} p_{2} p_{3}$.

An edge $u v$ is called constrained Gabriel edge (or simply, Gabriel edge here) if $\|u v\| \leq 1$ and the open disk using $u v$ as diameter does not contain any node from $V$. It is well-known [23] that the constrained Gabriel graph is a subgraph of the Delaunay triangulation, more precisely, $G G(V) \subseteq U \operatorname{Del}(V)$. Recall that a triangle $\triangle u v w$ belongs to the Delaunay triangulation $\operatorname{Del}(V)$ if its circumcircle $\operatorname{disk}(u, v, w)$ does not contain any other node of $V$ in its interior. To simplify the proofs, from now on, we assume that there are no four nodes of $V$ cocircumcircle. If four nodes are on the same circle, a very small random perturbation to their coordinates allows the assumption above without causing any problems in the actual network. It is easy to show that nodes $u, v$, and $w$ together cannot decide if they can form a triangle $\Delta u v w$ in $\operatorname{Del}(V)$ by using only their local information. We say a node $x$ can see another node $y$ if $\|x y\| \leq 1$. The following definition is one of the key ingredients of our localized algorithm.

Definition 1. A triangle $\triangle$ uvw satisfies $k$-localized Delaunay property if the interior of the circumcircle disk $(u, v, w)$ does not contain any node of $V$ that is a k-neighbor of $u, v$, or w; and all edges of the triangle $\triangle$ uvw have a length of no more than one unit. Triangle $\triangle$ uvw is called a $k$-localized Delaunay triangle.

Definition 2. The $k$-localized Delaunay graph over a node set $V$, denoted by $L D e l^{(k)}(V)$, has exactly all Gabriel edges and the edges of all k-localized Delaunay triangles.

When it is clear from the context, we will omit the integer $k$ in our notation of $L D e l^{(k)}(V)$. Our original conjecture was that $L \operatorname{Del}^{(1)}(V)$ is a planar graph and, thus, we can easily construct a planar $t$-spanner of $U D G(V)$ by using a localized approach. Unfortunately, as we will show later, the edges of the graph $L \operatorname{Del}^{(1)}(V)$ may intersect. While $L D e l^{(1)}(V)$ is a $t$-spanner, its construction is a little bit more complicated than some other nonplanar $t$-spanners, such as the Yao structure [21] and the $\theta$-graph [22]. But, we can make $L D e l^{(1)}(V)$ planar efficiently, a result we describe later in this paper.

The $k$-localized Delaunay graph $L D e l^{(k)}(V)$ over a node set $V$ satisfies a monotone property: $L \operatorname{Del}^{(k+1)}(V)$ is always a subgraph of $L D e l^{(k)}(V)$ for any positive integer $k$.

\section{1 $L D e l^{(k)}(V)$ is a $\boldsymbol{t}$-Spanner}

The following lemma was proven by us in [16]. 


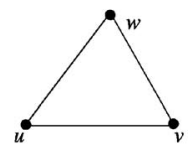

Case 1

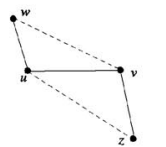

Case 2.1

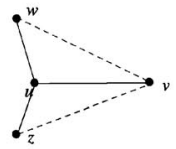

Case 2.2

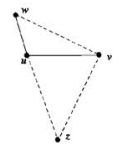

Case 3

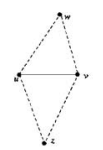

Case 4

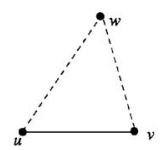

Case 5.1

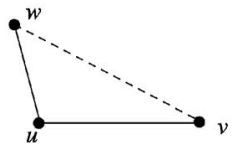

Case 5.2

Fig. 1. The neighborhood configuration of edge $u v$. Dashed lines (solid lines) denote edges with length $>1(\leq 1)$.

Lemma 1. Graph $U \operatorname{Del}(V)$ is a t-spanner of $U D G(V)$ for $t \leq 2.42$.

Theorem 1. Graph $U \operatorname{Del}(V)$ is a subgraph of the k-localized Delaunay graph $\operatorname{LDel}^{(k)}(V)$.

Proof. We prove the theorem by showing that each edge $u v$ of the unit Delaunay triangulation graph $U \operatorname{Del}(V)$ appears in the localized Delaunay graph $\operatorname{LDel}^{(k)}(V)$. For each edge $u v$ of $U \operatorname{Del}(V)$, the following five cases are possible (see Fig. 1 for illustrations).

Case 1: There is a triangle $\triangle u v w$ incident on $u v$ such that all edges of $\triangle u v w$ have length at most one unit. Because the circumcircle $\operatorname{disk}(u, v, w)$ is empty of nodes of $V$, triangle $\triangle u v w$ satisfies the $k$-localized Delaunay property and, thus, edge $u v$ belongs to $L D e l^{(k)}(V)$.

Case 2: Each of the two triangles incident on $u v$ has only one edge longer than one unit.

Case 3: One triangle $\triangle u v w$ incident on $u v$ has only one edge with length larger than one unit and the other triangle $\triangle u v z$ has two edges with length larger than one unit.

Case 4: Each of the two triangles incident on $u v$ has two edges longer than one unit.

We prove Cases 2, 3, and 4 together. Assume the two triangles are $\triangle u v w$ and $\triangle u v z$. Let $H_{u v, w}$ be the half-plane that is divided by $u v$ and contains node $w$. Then, edge $u v$ is not the longest edge in triangle $\triangle u v w$ and, thus, the angle $\angle u w v<\frac{\pi}{2}$. This implies that the circumcircle $\operatorname{disk}(u, v, w)$ contains $\operatorname{disk}(u, v) \cap H_{u v, w}$. Similarly, the other half of $\operatorname{disk}(u, v)$ is contained inside the circumcircle $\operatorname{disk}(u, v, z)$. Both $\operatorname{disk}(u, v, w)$ and $\operatorname{disk}(u, v, z)$ do not contain any node of $V$ inside. It implies that $\operatorname{disk}(u, v)$ is empty, i.e., edge $u v$ is a Gabriel edge. Consequently, edge $u v$ will be inserted to $L_{D e l}^{(k)}(V)$.

Case 5: There is only one triangle incident on $u v$ and it has at least one edge with length larger than one unit. Similar to Cases 2,3, and 4, we can show that $\operatorname{disk}(u, v)$ is empty and, therefore, edge $u v$ will be inserted to $L_{D e l}^{(k)}(V)$ as a Gabriel edge.

\section{2 $\operatorname{LDel}^{(1)}(V)$ May Be Nonplanar}

The definition of the 1-localized Delaunay triangle does not prevent two triangles from intersecting or prevent a Gabriel edge from intersecting a triangle. Fig. 2 gives such an example with six nodes $\{u, v, w, x, y, z\}$ that $L \operatorname{Del}^{(1)}(V)$ is not a planar graph. Here, $\|u v\|=\|x y\|=1$, and $\|u y\|=\|v y\|>1$. Node $x$ is out of circumcircle of $\operatorname{disk}(u, v, w)$. Triangle $\triangle u v w$ is a 1-localized Delaunay triangle. If the node $z$ does not exist, edge $x y$ is a Gabriel edge. The triangle $\triangle u v w$ intersects the Gabriel edge $x y$ if $z$ does not exist, otherwise, it intersects the 1-localized Delaunay triangle $\triangle x y z$. The example illustrated by Fig. 2 also implies that a triangle in $\operatorname{LDel}^{(1)}(V)$ can intersect many other edges (by creating equal-length Gabriel edges $x_{1} y_{1}, x_{2} y_{2}, \cdots$, which are parallel to Gabriel edge $x y$ ).

\section{3 $\operatorname{LDel}^{(1)}(V)$ Has Thickness 2}

In this section, we claim that $L D e l^{(1)}(V)$ has thickness two, or, in other words, its edges can be partitioned in two planar graphs. From Euler's formula, it follows that a simple planar graph with $n$ nodes has at most $3 n-6$ edges and, therefore, $L D e l^{(1)}(V)$ has at most $6 n$ edges. The proof of the following theorem is in the Appendix.

Theorem 2. Graph $\operatorname{LDel}^{(1)}(V)$ has thickness 2.

Our construction algorithm of $\operatorname{LDel}^{(1)}(V)$ below also implies that $\operatorname{LDel}^{(1)}(V)$ has a linear number of edges. Theorem 2 gives a better constant.

\section{4 $\operatorname{LDel}^{(k)}(V), k \geq 2$, Is Planar}

Although we gave an example showing that $\operatorname{LDel}^{(1)}(V)$ is not always a planar graph, we will show that $L_{D e l}^{(k)}(V)$, $k \geq 2$, is always planar. Theorem 1 implies that each edge $u v$ of $U \operatorname{Del}(V)$ is either a Gabriel edge or forms a 1-localized Delaunay triangle with some edges from $U \operatorname{Del}(V)$. Obviously, any two edges in $U \operatorname{Del}(V)$ do not intersect. Thus, each possible intersection in $\operatorname{LDel}^{(k)}(V)$ is caused by at least one edge of some localized Delaunay triangle. We begin the proof that $\operatorname{LDel}^{(k)}(V), k \geq 2$, is planar by giving some simple facts and lemmas.

Remark. If a Gabriel edge $u v$ intersects an edge $x y$, then $x y$ does not belong to $U \operatorname{Del}(V)$.

Lemma 2. If a Gabriel edge uw intersects a localized Delaunay triangle $\triangle x y z$, then $u$ and $w$ cannot be both outside the circumcircle disk $(x, y, z)$.

Proof. Let $c$ be the circumcenter of the triangle $\triangle x y z$. Then, at least one of the $x, y$, and $z$ must be on the different side of line $u w$ versus the center $c$; say $x$. If both $u$ and $w$ are outside the circumcircle $\operatorname{disk}(x, y, z)$, then $\left\langle w x u>\frac{\pi}{2}\right.$.

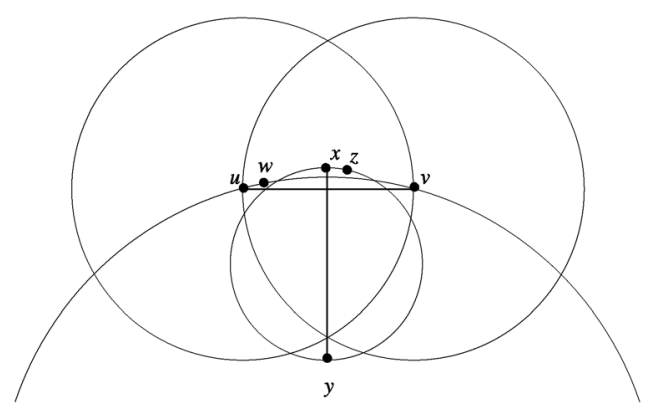

Fig. 2. $\operatorname{LDel}^{(1)}(V)$ is not planar. 
Thus, $x$ is inside $\operatorname{disk}(u, w)$, which contradicts that $u w$ is a Gabriel edge.

Lemma 3. Let $\triangle u v w$ and $\triangle x y z$ be two triangles of $L_{D e l}^{(k)}(V)$, $k \geq 1$, and assume the edge uw intersects the triangle $\triangle x y z$ and that $\operatorname{disk}(u, v, w)$ does not contain any of the nodes of $\{x, y, z\}$. Then, $\operatorname{disk}(x, y, z)$ contains either $u$ or $w$.

See the Appendix for the proof. The above lemma guarantees that, if two $k$-localized Delaunay triangles $\triangle u v w$ and $\triangle x y z$ intersect, then either $\operatorname{disk}(u, v, w)$ or $\operatorname{disk}(x, y, z)$ violates the Delaunay property by just considering the nodes $\{u, v, w, x, y, z\}$.

The following theorem gives more insight regarding localized Delaunay graphs and it is never used later in the paper. Its proof is immediate from Theorem 5 .

Theorem 3. $\operatorname{LDel}^{(2)}(V)$ is a planar graph.

In conclusion, we defined a sequence of localized Delaunay graphs $L D e l^{(k)}(V)$, where $1 \leq k \leq n$. All graphs are $t$-spaner of the unit-disk graph with the following properties:

- $U \operatorname{Del}(V) \subseteq L \operatorname{Del}^{(k)}(V)$, for all $1 \leq k \leq n$.

- $\quad L \operatorname{Del}^{(k+1)}(V) \subseteq \operatorname{LDel}^{(k)}(V)$, for all $1 \leq k \leq n$.

- $\quad L D e l^{(k)}(V)$ are planar graphs for all $2 \leq k \leq n$.

- $\quad L D e l^{(1)}(V)$ is not always planar.

\section{Localized Algorithm}

In this section, we study how to locally construct a planar $t$-spanner of $U D G(V)$. We use $q$ to denote the number of bits required to represent the unique node IDs and $p$ to denote the number of bits required to represent the geometric position of a node. When using big-Oh notation, we make the reasonable assumption that both $q$ and $p$ are $O(\log n)$.

Although the graph $U \operatorname{Del}(V)$ is a $t$-spanner for $U D G(V)$, it cannot be constructed locally. We can construct $\operatorname{LDel}^{(2)}(V)$, which is guaranteed to be a planar spanner of $U \operatorname{Del}(V)$, but the total communication cost of this approach could be $O(m \log n)$ bits, where $m$ is the number of edges in $U D G(V)$ and could be as large as $O\left(n^{2}\right)$. In order to reduce the total communication cost to $O(n \log n)$ bits, we do not construct $L \operatorname{Del}^{(2)}(V)$ and, instead, we extract a planar graph $P L \operatorname{Del}(V)$ out of $\operatorname{LDel}^{(1)}(V)$.

\subsection{Algorithm}

Recall that $\operatorname{LDel}^{(1)}(V)$ is not guaranteed to be a planar graph. Our algorithm first constructs $L D e l^{(1)}(V)$ and then removes edges from it to make it planar. The result of the algorithm is called $P \operatorname{LDel}(V)$ and we show later that it is planar and that it contains $U \operatorname{Del}(V)$ as a subgraph (and, therefore, it is a $t$-spanner of $U D G(V))$.

We assume that when a node sends out a message, all neighboring nodes will receive this message immediately.

\section{Algorithm 1: Localized Unit Delaunay Triangulation}

1. Each wireless node $u$ broadcasts an announce message with its identity and location to its neighbors $N_{1}(u)$ and listens to the messages from other nodes in $N_{1}(u)$.
2. Assume that node $u$ gathered the location information of $N_{1}(u)$. It computes the Delaunay triangulation $\operatorname{Del}\left(N_{1}(u)\right)$ of its 1-neighbors $N_{1}(u)$, including $u$ itself.

3. For each edge $u v$ of $\operatorname{Del}\left(N_{1}(u)\right)$, let $\triangle u v w$ and $\triangle u v z$ be two triangles incident on $u v$. Edge $u v$ is a Gabriel edge if both angles $\angle u w v$ and $\angle u z v$ are less than $\pi / 2$. Node $u$ marks all Gabriel edges $u v$, which will never be deleted.

4. Each node $u$ finds all triangles $\triangle u v w$ from $\operatorname{Del}\left(N_{1}(u)\right)$ such that $\|v w\| \leq 1$ and $\angle w u v \geq \frac{\pi}{3}$. The node sorts the edges $u v \in \operatorname{Del}\left(N_{1}(u)\right)$ in clockwise order and broadcast a proposal message. The proposal message contains $u$ 's ID, followed by the IDs of the ordered nodes $v$ with $u v \in \operatorname{Del}\left(N_{1}(u)\right)$. A single bit sent in between two IDs of $v$ and $w$ indicates if $\triangle u v w$ is as above, and we say that $u$ is proposing that $\triangle u v w$ be added to $\operatorname{LDel}^{(1)}(V)$. Then, node $u$ listens to the messages from other nodes in $N_{1}(u)$.

5. Each node $u$, after receiving a proposal message from node $v$, computes if $v$ is proposing adding triangles $\triangle u v w$ and $\triangle u v z$ to $L_{D e l}(1)(V)$. Then, $u$ computes if any of the triangles proposed by other nodes belongs to $\operatorname{Del}\left(N_{1}(u)\right)$. After computing all the triangles, $u$ broadcast an accept message which contains the IDs of $u$, the clockwise list of vertices $v$ such that $u$ computed that for some $w$ the triangle $\triangle u v w$ belongs to $\operatorname{Del}\left(N_{1}(u)\right)$, and a bit indicating if two such consecutive $v, v^{\prime}$ form a triangle $\triangle u v v^{\prime}$ of $\operatorname{Del}\left(N_{1}(u)\right)$.

6. A node $u$ adds the edges $u v$ and $u w$ to its set of incident edges if the triangle $\triangle u v w$ is in $\operatorname{Del}\left(N_{1}(u)\right)$ and both $v$ and $w$ have sent either a propose message or an accept message for triangle $\triangle u v w$.

It turns out (see below) that the edges added by all the vertices in this step form $\operatorname{LDel}^{(1)}(V)$. At this moment, the planarization phase starts.

7. Each node $u$ broadcasts a check message with its ID and the position of all vertices $v$ such that $u v \in \operatorname{LDel}^{(1)}(V)$.

8. Based on the check messages sent by the nodes in $N_{1}(u), u$ computes for every 1-local Delaunay triangle $\triangle u v w$ if any node is inside the circumcircle of $\triangle u v w$. If such a node is found, $u$ discards the triangle $\triangle u v w$. Then, the node $u$ sorts clockwise the edges $u v$ which are either Gabriel or belong to a triangle of $\operatorname{LDel}^{(1)}(V)$ which was not discarded.

9. Each node $u$ broadcasts an alive message, consisting of its ID, followed by the IDs of nodes incident to $u$ and a single bit in between two such nodes $v$ and $w$ telling if $\triangle u v w \in \operatorname{LDel}^{(1)}(V)$ and $\triangle u v w$ was not discarded.

10. Node $u$ keeps the edge $u v$ in its set of incident edges if it is a Gabriel edge, or if there is a triangle $\triangle u v w \in$ $L_{D e l}^{(1)}(V)$ which appears in the alive messages of $u$, $v$, and $w$. This implicitly creates the graph $P L \operatorname{Del}(V)$ and finishes the algorithm.

We first claim that the graph constructed at the end of Step 6 of the above algorithm is $L D e l^{(1)}(V)$. Indeed, for each triangle $\triangle u v w$ of $\operatorname{LDel}^{(1)}(V)$, one of its interior angle is at least $\pi / 3$ and $\triangle u v w$ is in $\operatorname{Del}\left(N_{1}(u)\right), \operatorname{Del}\left(N_{1}(v)\right)$, and 
$\operatorname{Del}\left(N_{1}(w)\right)$. So, one of the nodes among $\{u, v, w\}$ will broadcast the message proposal to form a 1-localized Delaunay triangle $\triangle u v w$ and the other two nodes will accept the proposal. Thus, $L D e l^{(1)}(V)$ is a subgraph of the constructed graph. Obviously, the constructed graph is also a subgraph of $\operatorname{LDel}^{(1)}(V)$ by definition, which in turn implies that they are the same.

Second, we show that $P \operatorname{LDel}(V)$ is indeed a planar graph.

\section{Theorem 4. $P L \operatorname{Del}(V)$ is a planar graph.}

Proof. Two Gabriel edges do not intersect. Then, every intersection must involve a localized Delaunay triangle $\triangle x y z$ which was broadcast alive by all three $x, y$, and $z$.

Assume that an edge $u w$ intersects a 1-localized Delaunay triangle $\triangle x y z$ on an edge $x y$. Edge $u w$ is either a Gabriel edge or an edge of another 1-localized Delaunay triangle, say $\triangle u v w$. In either case, either Lemma 2 or Lemma 3 implies that either $u$ or $w$ is inside the $\operatorname{disk}(x, y, z)$. By symmetry, we assume $w$ is inside the $\operatorname{disk}(x, y, z)$. The triangle inequality implies that

$$
\|u x\|+\|w y\|<\|u w\|+\|x y\| \leq 2 .
$$

The fact that triangle $\triangle x y z$ is in $P L D e l(V)$ implies that $w \notin N_{1}(x) \cup N_{1}(y) \cup N_{1}(z)$. Thus, $\|w y\|>1$, which implies that $\|u x\|<1$. In other words, $u \in N_{1}(x)$.

When $u$ broadcasts its check message, $x$ does find out the existence of a node inside $\operatorname{disk}(x, y, z)$. Then, $x$ does not broadcast $\triangle x y z$ in its alive message. We obtained a contradiction, thus completing the proof.

Note that any triangle of $\operatorname{LDel}^{(1)}(V)$ not kept by the algorithm is not a triangle of $\operatorname{LDel}^{(2)}(V)$. Therefore, we have:

Theorem 5. $P L D e l(V)$ is a supergraph of $\operatorname{LDel}^{(2)}(V)$.

Next, we carefully analyze the communication cost of the algorithm. There are five types of messages and each includes a few bits to describe which type. Disregarding the few other bits used by every message, we have:

1. announce messages contain in total $n(p+q)$ bits, and

2. proposal messages contain in total $11 n q$ bits. Indeed, if the proposal of a node includes the nodes $v_{1}, v_{2}, \ldots, v_{j}$, then for every $1 \leq i \leq j$, either $\angle v_{i} u v_{i+1} \geq \frac{\pi}{3}$ or $\angle v_{i-1} u v_{i} \geq \frac{\pi}{3}$ (where, for convenience, we assume $v_{0}=v_{j}$ and $\left.v_{1}=v_{j+1}\right)$. Immediate geometric arguments imply that $j \leq 10$.

3. Each accept message contains the triangle incident with some vertex $u$. Those triangles are either "big" or "small" as defined in the proof of Theorem 2. The graph given by big triangles and the graph given by small triangles are planar. Using Euler's formula, we obtain that there are at most $4 n$ triangles accepted by two nodes. In total, at most $6 n$ triangles are implicitly proposed. Counting multiplicity (some proposed triangles are accepted once and some are accepted twice), a total of $10 n$ triangles are accepted-each contributing one ID to the communication cost. As each accept message also contains the ID of the sender, a total of at most $11 n$ IDs are broadcast by accept messages.

4. Each check message contains the ID of a node $u$ and a number of positions corresponding to $u$ 's neighbors in $L \operatorname{Del}^{(1)}(V)$. As $L \operatorname{Del}^{(1)}(V)$ has a thickness of two, it has at most $6 n$ edges and, therefore, a total of at most $12 n$ positions are broadcast in check messages.

5. Each alive message contains the ID of a node $u$ and a number IDs corresponding to $u$ 's neighbors in $\mathrm{LDel}^{(1)}(V)$, and bits indicating whether consecutive neighbors make a triangle not yet discarded. As shown above, there are at most $n+12 n=13 n$ IDs broadcast.

From the previous discussion, Theorem 1, Theorem 2, and the results of [16], [17] regarding the spanning ratio of $U \operatorname{Del}(V)$, we obtain:

Theorem 6. $P L D e l(V)$ is planar $\frac{4 \sqrt{3}}{9} \pi$-spanner of $U D G(V)$, and can be constructed with total communication cost of $n(37 q+13 p+100)$ bits, where $q$ is the number of bits required to represent a node $I D, p$ is the number of bits required to represent the position of a node, and 100 bits are used to represent all messages types.

Recently, Calinescu [35] presented a localized method such that all wireless nodes collectively find the 2-hop neighbors $N_{2}(u)$ for every node $u$ with $O(n \log n)$ communication complexity with the assumption that the geometric location of every node is known. The knowledge of the 2-hop neighbors information allows the direct construction of $\mathrm{LDel}^{2}(V)$. However, the hidden constant of [35] is much larger than the constant presented here.

\section{RoUting}

In this section, we discuss how to route packets on the constructed graph. Recently, Bose and Morin [9] first proposed a localized routing algorithm that routes a packet using the Delaunay triangulation and guarantees the distance traveled by the packet is no more than a small constant factor of the distance between the source and the destination nodes. However, this algorithm has a major deficiency by requiring the construction of the Delaunay triangulation and the Voronoi diagram of all wireless nodes, which could be very expensive in distributed computing. Bose et al. [11] also proposed another method, called Face routing, that routes the packets using the Gabriel graph to guarantee the delivery. The Gabriel graph is a subgraph of $P \operatorname{LDel}(V)$. Thus, if we apply the routing method proposed in [11] on the newly proposed planar graph $P L D e l(V)$, we expect to achieve better performance because $P L \operatorname{Del}(V)$ is denser than the Gabriel graph (but, still with $O(n)$ edges). The constructed local Delaunay triangulation not only guarantees that the length of the shortest path connecting any two wireless nodes is at most a constant factor of the minimum in the unit-disk graph, but it also guarantees that the energy consumed by the path is also minimum, as it includes the Gabriel graph (see [36], [27]). Moreover, because the constructed topology is planar, a localized routing algorithm using the right-hand rule guarantees the delivery of the packets from source node to the destination node. 

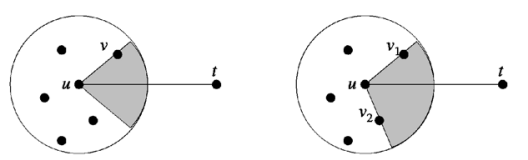

Compass

Random Compass
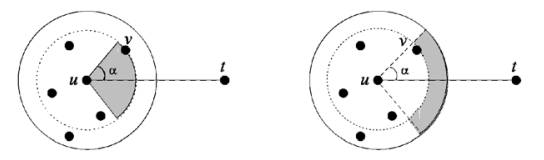

Most Forwarding Nearest Neighbor Farthest Neighbor

Fig. 3. Shaded area is empty and $v$ is next node.

We study the following routing algorithms, illustrated by Fig. 3, on the graphs proposed in this paper.

Compass Routing ( $\mathrm{Cmp}$ ) Let $t$ be the destination node. Current node $u$ finds the next relay node $v$ such that the angle $\angle v u t$ is the smallest among all neighbors of $u$ in a given topology. See [20].

Random Compass Routing (RndCmp) Let $u$ be the current node and $t$ be the destination node. Let $v_{1}$ be the node on the above of line $u t$ such that $\angle v_{1} u t$ is the smallest among all such neighbors of $u$. Similarly, we define $v_{2}$ to be nodes below line $u t$ that minimizes the angle $\angle v_{2} u t$. Then, node $u$ randomly chooses $v_{1}$ or $v_{2}$ to forward the packet. See [20].

Greedy Routing (Grdy) Let $t$ be the destination node. Current node $u$ finds the next relay node $v$ such that the distance $\|v t\|$ is the smallest among all neighbors of $u$ in a given topology. See [11].

Most Forwarding Routing (MFR) Current node $u$ finds the next relay node $v$ such that $\left\|v^{\prime} t\right\|$ is the smallest among all neighbors of $u$ in a given topology, where $v^{\prime}$ is the projection of $v$ on segment $u t$. See [10].

Nearest Neighbor Routing (NN) Given a parameter angle $\alpha$, node $u$ finds the nearest node $v$ as forwarding node among all neighbors of $u$ in a given topology such that $\angle v u t \leq \alpha$.

Farthest Neighbor Routing (FN) Given a parameter angle $\alpha$, node $u$ finds the farthest node $v$ as forwarding node among all neighbors of $u$ in a given topology such that $\langle v u t \leq \alpha$.

It is shown in [11], [20] that the compass routing, random compass routing, and the greedy routing guarantee to deliver the packets from the source to the destination if Delaunay triangulation is used as network topology. They proved this by showing that the distance from the selected forwarding node $v$ to the destination node $t$ is less than the distance from current node $u$ to $t$. However, the same proof cannot be carried over when the network topology is Yao graph, Gabriel graph, relative neighborhood graph, and the localized Delaunay triangulation.

We present our experimental results of various routing methods on different network topologies. Fig. 4 illustrates some network topologies discussed in this paper. Recall that Gabriel graph, relative neighborhood graph, Delaunay triangulation, $\operatorname{LDel}^{(2)}(V)$, and $P L D e l(V)$ are always planar graphs. The Yao structure, Delaunay triangulation, $\operatorname{LDel}^{(2)}(V)$, and $P L \operatorname{Del}(V)$ are always a $t$-spanner of the unit-disk graph. We use integer parameter $k=8$ in constructing the Yao graph. In the experimental results presented here, we choose total $n=50$ wireless nodes which are distributed randomly in a square area with side length 100 meters. Each node is specified by a random $x$-coordinate value and a random $y$-coordinate value. The transmission radius of each wireless node is set as 30 meters. We randomly select 10 percent of nodes as source nodes and, for every source node, we randomly choose 10 percent of nodes as destination nodes. The statistics are computed over 10 different node configurations. Interestingly, we found that, when the underlying network topology is Yao graph, $\operatorname{LDel}^{(2)}(V)$, or $\operatorname{PLDel}(V)$, the compass routing, random compass routing, and the greedy routing delivered the packets in all our experiments. Table 1 illustrates the delivery rates of different localized routing protocols on various topologies. For nearest neighbor routing and farthest neighbor routing, we choose the angle $\alpha=\pi / 3$. The $\operatorname{LDel}^{(2)}(V)$ and $P L \operatorname{Del}(V)$ graphs be preferred over the Yao graph because we can apply the right-hand rule when previous simple heuristic localized routing fails. The reason that the compass, random compass, and greedy methods are able to guarantee the delivery of the packets in our simulations may be as follows: When the transmission range of a node is large enough, the localized Delaunay triangulation of a randomly uniformly distributed point set is almost the same as the Delaunay triangulation (this can be proven [37]). We already know that these three methods guarantee delivery if Delaunay triangulation is used. RNG and GG have small delivery rate when these simple localized heuristics are used since these two structures have less edges than other structures and, therefore, each node often has less choices of nodes to relay messages. Table 2 illustrates the maximum spanning ratios achieved by different routing methods on various topologies. In our experiment, these ratios are considerably small.

We also conducted extensive simulations of the Face routing method [11] and the Greedy-Face-Greedy (GFG)

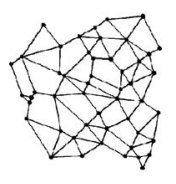

GG

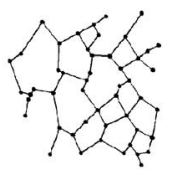

RNG

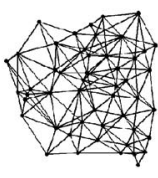

Yao

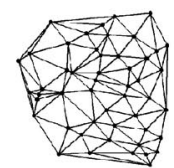

Del

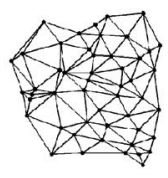

$\mathrm{LDel}^{(2)}$

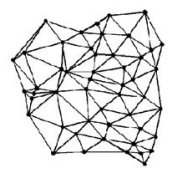

PLDel

Fig. 4. Various planar network topologies (except Yao). 
TABLE 1

The Delivery Rate of Different Localized Routing Methods on Various Network Topologies

\begin{tabular}{|l|c|c|c|c|c|c|}
\hline & Yao & RNG & GG & Del & LDel $^{(2)}$ & PLDel \\
\hline \hline NN & 98.7 & 44.9 & 83.2 & 99.1 & 97.8 & 98.3 \\
\hline FN & 97.5 & 49 & 81.7 & 92.1 & 97 & 97.6 \\
\hline MFR & 98.5 & 78.5 & 96.6 & 95.2 & 96.6 & 99.7 \\
\hline Cmp & 100 & 86.6 & 99.6 & 100 & 100 & 100 \\
\hline RndCmp & 100 & 91.7 & 99.9 & 100 & 100 & 100 \\
\hline Grdy & 100 & 87.5 & 99.6 & 100 & 100 & 100 \\
\hline
\end{tabular}

routing method [38] on the Gabriel graph and the local Delaunay triangulation $\operatorname{LDel}^{1}(V)$. We choose $n=$ $20,30, \cdots, 90,100$ nodes randomly and uniformly distributed in a square of length 100 meters. The uniform transmission range of nodes are set as $r$, where $r$ varies from $30,40,50,60$, or 70 meters. Since it will be hard to control the network density directly, we change the network density by indirectly changing the number of nodes in the network or the transmission range of the nodes. A large number of nodes or larger transmission range will result in a denser network. See Tables 3 and 4 . For a given point set, we randomly select 10 percent of nodes as sources and 10 percent of nodes as targets. The maximum and average spanning ratio is computed for all chosen pair of nodes. Given $n$ and $r$, we generate 10 sets of random $n$ points. We found that the spanning ratio of the Face routing method is significantly smaller when the local Delaunay triangulation is used instead of the Gabriel graph. This may be due to the fact that local Delaunay triangulation has more edges, thus the number of faces traversed by the Face routing algorithm is often smaller when LDEL is used than when GG is used.

The average spanning ratios of the GFG method on the Gabriel graph and the local Delaunay triangulation are
TABLE 2

The Maximum Spanning Ratio of Different Localized Routing Methods on Various Network Topologies

\begin{tabular}{|l|c|c|c|c|c|c|}
\hline & Yao & RNG & GG & Del & LDel $^{(2)}$ & PLDel \\
\hline \hline NN & 1.9 & 2.1 & 1.9 & 1.7 & 1.8 & 1.9 \\
\hline FN & 4.2 & 2.8 & 2.7 & 5.2 & 3.4 & 3.1 \\
\hline MFR & 4.8 & 3.2 & 2.4 & 4.5 & 3.9 & 4.1 \\
\hline Cmp & 3.3 & 2.9 & 2.8 & 1.6 & 1.8 & 2.0 \\
\hline RndCmp & 2.7 & 3.0 & 2.4 & 1.7 & 2.0 & 1.8 \\
\hline Grdy & 2.1 & 3.5 & 2.2 & 2.0 & 1.9 & 1.9 \\
\hline
\end{tabular}

similar when the number of nodes in the network is no more than 100. We expect our local Delaunay triangulation to perform better when the number of nodes are significantly large since, the spanning ratio of the Gabriel graph on a set of $n$ random points is about $\Omega(\sqrt{\log n / \log \log n})$. When $n$ is small, $\Omega(\sqrt{\log n / \log \log n})$ is almost a small constant, but when $n$ is large, $\Omega(\sqrt{\log n / \log \log n})$ cannot be treated as a small constant anymore. Notice that our local Delaunay triangulation guarantees that there is always a path to connect any two nodes with length no more than 2.5 times the length of the shortest path connecting them in the original UDG.

\section{CONCLUSION}

It is well-known that Delaunay triangulation $\operatorname{Del}(V)$ is a $t$-spanner of the completed graph $K(V)$. In this paper, we defined several new structures and then gave a localized algorithm that constructs a graph, namely, $P L \operatorname{Del}(V)$. We proved that $P L D e l(V)$ is a planar graph and it is a $t$-spanner by showing that $U \operatorname{Del}(V)$ is a subgraph of $P L \operatorname{Del}(V)$. The total number of messages sent by all nodes in our algorithm is $O(n \log n)$ bits. Our experiments showed that the delivery

TABLE 3

The Maximum Spanning Ratio of Face (over the Fraction Line) and GFG (under the Fraction Line) Routing Methods on the Gabriel Graph and Local Delaunay Triangulation

\begin{tabular}{|c|c|c|c|c|c|}
\hline $\mathrm{n}$ & 0.3 & 0.4 & 0.5 & 0.6 & 0.7 \\
\hline 20 & $11.5 / 7.6$ & $15.4 / 4.9$ & $9.9 / 1.7$ & $18.4 / 1.6$ & $13.0 / 1.6$ \\
\hline 30 & $34.2 / 5.8$ & $17.9 / 5.6$ & $18.3 / 2.0$ & $14.7 / 2.0$ & $21.5 / 3.1$ \\
\hline 40 & $22.7 / 16.6$ & $21.1 / 9.3$ & $14.7 / 9.8$ & $16.2 / 4.6$ & $15.6 / 13.8$ \\
\hline 50 & $15.4 / 1.9$ & $20.6 / 1.8$ & $31.5 / 1.9$ & $14.6 / 1.6$ & $18.0 / 2.0$ \\
\hline 60 & $27.6 / 7.6$ & $17.3 / 1.6$ & $18.5 / 1.7$ & $19.1 / 14.2$ & $25.7 / 1.8$ \\
\hline 70 & $25.3 / 17.7$ & $30.4 / 9.9$ & $19.5 / 1.9$ & $22.0 / 2.6$ & $20.9 / 1.9$ \\
\hline 80 & $30.1 / 1.7$ & $49.4 / 1.6$ & $22.4 / 1.7$ & $20.8 / 6.5$ & $23.4 / 1.7$ \\
\hline 90 & $16.0 / 1.9$ & $33.7 / 2.8$ & $14.9 / 3.7$ & $32.2 / 10.4$ & $19.8 / 1.9$ \\
\hline 100 & $19.2 / 2.5$ & $43.8 / 3.5$ & $13.5 / 2.9$ & $54.6 / 3.9$ & $24.6 / 2.7$ \\
\hline
\end{tabular}

On Gabriel graph

\begin{tabular}{|c|c|c|c|c|c|}
\hline $\mathbf{n}$ & 0.3 & 0.4 & 0.5 & 0.6 & 0.7 \\
\hline 20 & $10.6 / 5.5$ & $7.8 / 1.6$ & $9.6 / 3.7$ & $13.2 / 1.6$ & $7.7 / 1.6$ \\
\hline 30 & $10.3 / 6.6$ & $14.2 / 4.5$ & $33.3 / 4.1$ & $14.2 / 2.2$ & $13.9 / 2.1$ \\
\hline 40 & $18.0 / 12.3$ & $24.1 / 2.2$ & $21.4 / 2.6$ & $30.5 / 10.3$ & $14.9 / 1.8$ \\
\hline 50 & $11.3 / 2.3$ & $21.6 / 1.8$ & $14.7 / 1.5$ & $23.2 / 2.1$ & $20.1 / 2.3$ \\
\hline 60 & $18.3 / 2.8$ & $21.9 / 1.9$ & $22.0 / 1.7$ & $16.2 / 2.2$ & $17.4 / 2.8$ \\
\hline 70 & $23.0 / 1.6$ & $60.2 / 1.8$ & $31.0 / 1.8$ & $19.0 / 1.9$ & $22.8 / 1.8$ \\
\hline 80 & $24.7 / 11.2$ & $24.4 / 2.2$ & $20.6 / 2.8$ & $33.9 / 2.2$ & $13.6 / 5.4$ \\
\hline 90 & $31.5 / 1.9$ & $20.1 / 2.2$ & $20.2 / 3.0$ & $52.8 / 2.0$ & $50.4 / 1.8$ \\
\hline 100 & $24.9 / 2.6$ & $11.1 / 4.4$ & $17.2 / 2.2$ & $16.7 / 9.2$ & $24.8 / 2.9$ \\
\hline
\end{tabular}

On local Delaunay triangulation 
TABLE 4

The Average Spanning Ratio of Face (over the Fraction Line) and GFG (under the Fraction Line) Routing Methods on the Gabriel Graph and Local Delaunay Triangulation

\begin{tabular}{|c|c|c|c|c|c|}
\hline $\mathrm{n}$ & 0.3 & 0.4 & 0.5 & 0.6 & 0.7 \\
\hline 20 & $3.0 / 1.8$ & $2.7 / 1.2$ & $2.7 / 1.2$ & $3.0 / 1.1$ & $2.4 / 1.2$ \\
\hline 30 & $3.4 / 1.3$ & $3.2 / 1.2$ & $3.7 / 1.2$ & $3.3 / 1.2$ & $3.6 / 1.2$ \\
\hline 40 & $4.2 / 1.2$ & $3.8 / 1.2$ & $3.7 / 1.3$ & $3.6 / 1.2$ & $3.7 / 1.3$ \\
\hline 50 & $4.4 / 1.2$ & $4.3 / 1.2$ & $4.6 / 1.2$ & $3.8 / 1.2$ & $4.0 / 1.2$ \\
\hline 60 & $4.6 / 1.2$ & $4.4 / 1.2$ & $4.5 / 1.2$ & $4.6 / 1.3$ & $4.6 / 1.2$ \\
\hline 70 & $5.2 / 1.2$ & $4.9 / 1.2$ & $4.9 / 1.2$ & $5.0 / 1.2$ & $5.0 / 1.2$ \\
\hline 80 & $5.1 / 1.2$ & $5.5 / 1.2$ & $5.7 / 1.2$ & $5.1 / 1.2$ & $5.3 / 1.2$ \\
\hline 90 & $5.0 / 1.2$ & $4.9 / 1.2$ & $4.7 / 1.3$ & $5.2 / 1.2$ & $5.5 / 1.2$ \\
\hline 100 & $5.2 / 1.2$ & $5.8 / 1.2$ & $6.3 / 1.2$ & $5.8 / 1.3$ & $5.6 / 1.2$ \\
\hline
\end{tabular}

On Gabriel graph

rates of existing localized routing protocols are increased when localized Delaunay triangulation is used instead of several previously proposed planar topologies. Our simulations also shows that the traveled distance of the packets is significantly less when the FACE routing algorithm or GFG routing is applied on LDEL than applied on GG.

We proved that the shortest path in $P L \operatorname{Del}(V)$ connecting any two nodes $u$ and $v$ is at most a constant factor of the shortest path connecting $u$ and $v$ in UDG. It remains open designing a localized algorithm such that the path traversed by a packet from $u$ to $v$ has length within a constant of the shortest path connecting $u$ and $v$ in UDG.

Recently, Li et al. [39], [40] present a novel method to construct bounded degree planar spanner locally.

\section{APPENDIX}

We start this with the proof of Lemma 3, which we restate below:

Lemma 4. Let $\triangle u v w$ and $\triangle x y z$ be two triangles of $L D e l^{(k)}(V)$, $k \geq 1$, and assume the edge $x y$ intersects the triangle $\triangle u v w$ and that disk $(x, y, z)$ does not contain any of the nodes of $\{u, v, w\}$. Then, $\operatorname{disk}(u, v, w)$ contains either $x$ or $y$.

The following lemma is implicitly used in its proof.

Lemma 5. If an edge $x y$ intersects a localized Delaunay triangle $\triangle u v w$, then it intersects two edges of $\triangle u v w$.

Proof. If it intersects one edge of $\triangle u v w$, then either $x$ or $y$ must be inside $\triangle u v w$, say $x$. Then $x u<\max (u v, u w) \leq 1$, which contradicts that $\triangle u v w$ is a localized Delaunay triangle.

We present some other useful facts and lemmas.

\begin{tabular}{|c|c|c|c|c|c|}
\hline $\mathrm{n}$ & 0.3 & 0.4 & 0.5 & 0.6 & 0.7 \\
\hline 20 & $2.9 / 1.3$ & $2.5 / 1.1$ & $3.0 / 1.1$ & $2.6 / 1.1$ & $2.2 / 1.1$ \\
\hline 30 & $3.2 / 1.2$ & $2.9 / 1.2$ & $3.5 / 1.2$ & $2.9 / 1.2$ & $3.4 / 1.2$ \\
\hline 40 & $3.9 / 1.2$ & $3.8 / 1.2$ & $4.2 / 1.2$ & $3.5 / 1.2$ & $4.2 / 1.2$ \\
\hline 50 & $3.3 / 1.2$ & $4.0 / 1.2$ & $4.2 / 1.2$ & $3.9 / 1.1$ & $4.0 / 1.2$ \\
\hline 60 & $4.4 / 1.2$ & $5.0 / 1.2$ & $4.7 / 1.2$ & $4.4 / 1.2$ & $4.4 / 1.2$ \\
\hline 70 & $4.9 / 1.1$ & $5.3 / 1.2$ & $5.5 / 1.2$ & $4.9 / 1.2$ & $5.0 / 1.2$ \\
\hline 80 & $5.2 / 1.2$ & $5.4 / 1.2$ & $5.1 / 1.2$ & $5.4 / 1.2$ & $5.0 / 1.2$ \\
\hline 90 & $5.5 / 1.2$ & $5.9 / 1.2$ & $5.6 / 1.2$ & $5.6 / 1.2$ & $5.3 / 1.2$ \\
\hline 100 & $6.1 / 1.3$ & $5.2 / 1.2$ & $6.0 / 1.2$ & $6.0 / 1.4$ & $6.4 / 1.2$ \\
\hline
\end{tabular}

On local Delaunay triangulation

Lemma 6. If an edge $x y$ intersects a localized Delaunay triangle $\triangle u v w$, then $x$ and $y$ can not be both inside the circumcircle $\operatorname{disk}(u, v, w)$.

Proof. For the sake of contradiction, assume that $x$ and $y$ are both inside $\operatorname{disk}(u, v, w)$. Fig. 5 illustrates the proof that follows. Notice that $\operatorname{disk}(u, v, w)$ is divided into four regions by the triangle $\Delta u v w$. Let $\widehat{u v}, \widehat{v w}$, and $\widehat{w u}$ be the three fan regions defined by edges $u v, v w$, and $w u$, respectively. First of all, neither $x$ nor $y$ can be inside the triangle $\triangle u v w$. Assume that $x$ is inside the region $\widehat{u v}$ and $y$ is inside the region $\widehat{v w}$. Then, one of the angles $\angle u w v$ and $\angle v u w$ is less than $\frac{\pi}{2}$, which implies that one of the angles $\angle u x v$ and $\angle v y w$ is larger than $\frac{\pi}{2}$. Thus, either $v y<$ $v w \leq 1$ or $v x<v u \leq 1$. In other words, the $\operatorname{disk}(u, v, w)$ contains a node from $N_{1}(v)$. This contradicts that $\triangle u v w$ is a $k$-localized Delaunay triangle.

We now present the proof of Lemma 3.

Proof. There are three cases: triangles $\triangle u v w$ and $\triangle x y z$ share two nodes (i.e., one edge), one node, or do not share any node.

Case 1: Triangles $\triangle u v w$ and $\triangle x y z$ share one edge.

Case 2: Triangles $\triangle u v w$ and $\triangle x y z$ share one node. These two cases are impossible. See the proof of

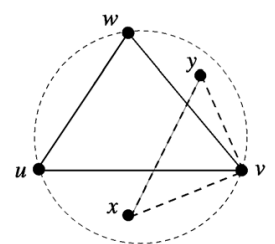

Fig. 5. Points $x, y$ cannot both be inside $\operatorname{disk}(u, v, w)$. 


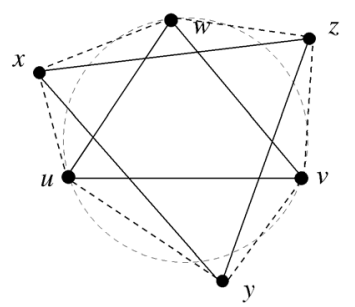

Subcase 3.1

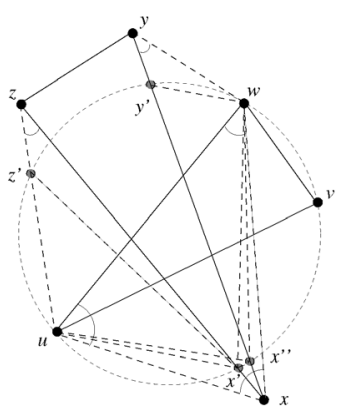

Subcase 3.2 (a)

(b)

Fig. 6. All or four edges of two triangles intersect.

Theorem 10 in the Appendix of our conference version [16].

Case 3: Triangles $\triangle u v w$ and $\triangle x y z$ do not share any node. It is not difficult to show that there are only two possible subcases as illustrated by Fig. 6 .

Subcase 3.1: All edges of $\triangle x y z$ and $\triangle u v w$ are intersected by some edges of the other triangle. Assume that the nodes have the order as illustrated by the Fig. 6a. Then, it is easy to show that all angles $\angle w x u, \angle x u y, \angle u y v, \angle y v z, \angle v z w$, and $\angle z w x$ are less than $\pi$. Notice that $\angle w x u+\angle w v u<\pi$ because $x$ is not inside the circumcircle $\operatorname{disk}(u, v, w)$. Similarly, $\angle u y v+\angle u w v<\pi$ and $\angle v z w+\angle v u w<\pi$. Therefore,

$\angle w x u+\angle u y v+\angle v z w<3 \pi-(\angle w v u+\angle u w v+\angle v u w)=2 \pi$.

Notice that

$\angle w x u+\angle u y v+\angle v z w+\angle x u y+\angle y v z+\angle z w x=4 \pi$.

It implies that $\angle x u y+\angle y v z+\angle z w x>2 \pi$. Then, we know that at least one of the nodes of $u, v$, and $w$ is contained inside the circumcircle $\operatorname{disk}(x, y, z)$ (otherwise by symmetry, similarly, we would have $\angle x u y+\angle y v z+\angle z w x<2 \pi)$. We prove that Subcase 3.1 is impossible. For the sake of contradiction, assume that it is possible. Then, from the proof of Subcase 3.1, either $\operatorname{disk}(u, v, w)$ contains one of the nodes of $x, y$, and $z$; or $\operatorname{disk}(x, y, z)$ contains at least one of the nodes of $u, v$, and $w$. Without loss of generality, assume that node $x$ is contained in the interior of $\operatorname{disk}(u, v, w)$. Then, Lemma 6 implies that both $y$ and $z$ are outside of $\operatorname{disk}(u, v, w)$. Fig. 7 illustrates the proof that follows. The existence of triangle $\triangle u v w$ implies that $\|x u\|>1$, $\|x v\|>1$, and $\|x w\|>1$. Notice that $\|x y\| \leq 1$ and $\|x z\| \leq 1$. Let $c$ be the circumcenter of the triangle $\triangle u v w$. Here, $c$ cannot be $x$ because $x u>1, x y \leq 1$, and $y$ is outside of the circle. The angle $\angle u x v<\frac{\pi}{3}$ because $u v$ must be the shortest edge of triangle $\triangle u x v$. Consider the following five segments lying in the interior of the wedge $u x v: x v, x z, x w, x y$, and $x u$. From the pigeonhole principle, there are at least three such segments lying on the same side of the line $x c$. More precisely, we have either $x v, x z$, and $x w$ on the same side of $x c$ or $x w, x y$, and $x u$ are on the same side of $x c$. Without loss of generality, assume that the first

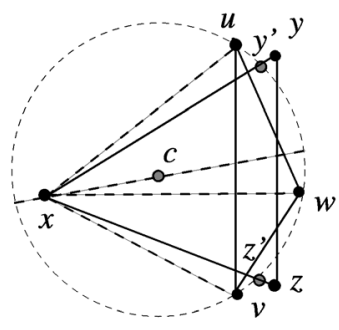

(a)

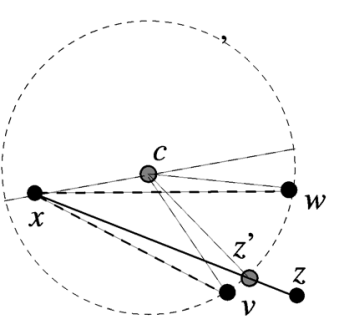

(b)
Fig. 7. Subcase 3.1 is impossible.

scenario happens. Then, it is easy to prove that $\|x z\|>\min (x v, x w)>1$. This contradicts to $\|x z\| \leq 1$. Fig. 7b illustrates the proof using that

$$
\|x v\|^{2}=\|x c\|^{2}+\|c v\|^{2}-2\|x c\| \cdot\|c v\| \cdot \cos (\angle x c v),
$$

and $\|c v\|=\left\|c z^{\prime}\right\|=\|c w\|$. Therefore, the assumption that Subcase 3.1 is possible does not hold.

Subcase 3.2: One edge of each triangle is not intersected by the edges of the other triangle. We prove that $\operatorname{disk}(x, y, z)$ contains at least one of the nodes of $u$ and $w$. Fig. $6 \mathrm{~b}$ illustrates the proof that follows. Let $x^{\prime}$ be the intersection point of segment $x z$ with the circumcircle $\operatorname{disk}(u, v, w)$, which is closer to $x$. Let $z^{\prime}$ be the intersection point of segment $u z$ with the circumcircle $\operatorname{disk}(u, v, w)$. Let $x^{\prime \prime}$ and $y^{\prime}$ be the two intersection points of segment $x y$ with the circumcircle $\operatorname{disk}(u, v, w)$, where $x^{\prime \prime}$ is closer to $x$ and $y^{\prime}$ is closer to $y$. Then,

$$
\begin{aligned}
& \angle x z u<\angle x^{\prime} z^{\prime} u=\angle x^{\prime} w u<\angle x w u, \text { and } \\
& \angle w y x<\angle w y^{\prime} x^{\prime \prime}=\angle w u x^{\prime \prime}<\angle w u x .
\end{aligned}
$$

Notice that $\angle y z u+\angle z u x+\angle u x w+\angle x w y+\angle w y z=3 \pi$. Then,

$(\angle y z x+\angle y w x)+(\angle z y x+\angle z u x)=3 \pi-(\angle x z u+\angle w y x+\angle u x w)$

$$
>3 \pi-(\angle x w u+\angle w u x+\angle u x w)=2 \pi .
$$

So, either $\angle y z x+\angle y w x>\pi$ or $\angle z y x+\angle z u x>\pi$ from the pigeonhole principle. Consequently, $\operatorname{disk}(x, y, z)$ contains either node $w$ or node $u$.

We present the proof that graph $L D e l^{(1)}(V)$ has a thickness of two.

Proof. We prove that $\operatorname{LDel}^{(1)}(V)$ has thickness two or, in other words, its edges can be partitioned in two planar graphs. Call a triangle $\triangle x y z$ of $\operatorname{LDel}^{(1)}(V)$ big if the radius of $\operatorname{disk}(u, v, w)$ is at least one, and small otherwise. A Gabriel edge can be thought as a small triangle, as if one of its endpoints is duplicated. Put all the Gabriel edges and the edges of small triangles in the graph $G_{1}$ and all the edges of the big triangles in $G_{2}$, possibly putting an edge in both $G_{1}$ and $G_{2}$ if it is shared by a small triangle and a big triangle. In the following, we show that both $G_{1}$ and $G_{2}$ are planar. More precisely, we prove that any intersection of two edges of $\operatorname{LDel}^{(1)}(V)$ involves a big triangle and a small triangle, or a big triangle and a Gabriel edge.

First, we show that the edges of two big triangles cannot intersect. Assume for a contradiction that $\triangle u v w$ 
(a)

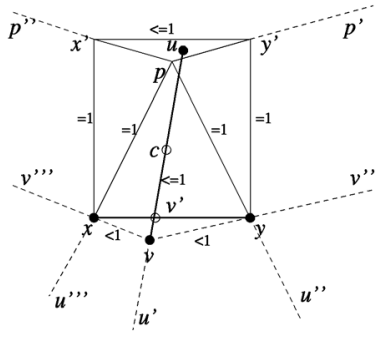

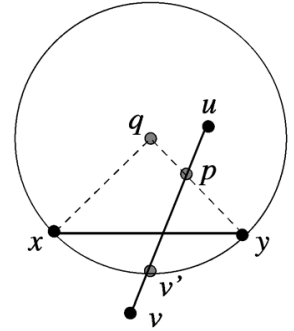

(b)
Fig. 8. (a) No two big triangles intersect. (b) No two small triangles intersect.

and $\triangle x y z$ are big triangles which intersect. We must be in Subcase 3.2 of the proof of Lemma 3. Rename the vertices of the triangles such that the following situation holds: $u$ is inside $\operatorname{disk}(x, y, z), v$ is outside $\operatorname{disk}(x, y, z)$, and the edges $u v$ and $x y$ intersect in the point $v^{\prime}$. Then, $\|u x\|>1$ and $\|u y\|>1$. Using the triangle inequality in $\triangle x v^{\prime} v$ and $\triangle u v^{\prime} y$, we obtain that $\|v x\|<1$ and, similarly, $\|v y\|<1$. Then, $\|q u\|=\|q v\| \geq 1$. Let $x^{\prime}$ and $y^{\prime}$ be the two points such that $x x^{\prime} y^{\prime} y$ is a rectangle with $\left\|x x^{\prime}\right\|=$ $\left\|y y^{\prime}\right\|=1$ such that $u$ and $x^{\prime}$ are on the same side of the line $x y$. Let $p$ be the point inside $x x^{\prime} y^{\prime} y$ such that $\|p x\|=\|p y\|=1$. Note that $u$ must be inside the rectangle $x x^{\prime} y^{\prime} y$. Indeed, otherwise, $1=\left\|x x^{\prime}\right\| \leq\left\|u v^{\prime}\right\|<1$. As $\left\|u v^{\prime}\right\|<1, u$ is inside the triangle $\triangle x^{\prime} y^{\prime} p$ or in the interior of the edge $x^{\prime} y^{\prime}$ because $u$ cannot be inside the triangle $\triangle x x^{\prime} p$ (otherwise, $\|u x\| \leq \max \left(\left\|x^{\prime} x\right\|,\|p x\|\right)=1$, which is impossible); similarly, $u$ cannot be inside the triangle $\triangle y y^{\prime} p$ and the triangle $\triangle x y p$. Let $q$ be the center of $\operatorname{disk}(u, v, w)$. Let $c$ be the midpoint of segment $u v$. Thus, $c q$ is a perpendicular bisector of segment $u v$. Then, $c$ must be between points $u$ and $v^{\prime}$. Indeed, otherwise, either $x$ or $y$ is on the same side of $c q$ with $u$, which contradicts to $\|u x\|>1 \geq\|v x\|$ and $\|u y\|>1 \geq\|v y\|$. We show that there is no place to put the circumcenter $q$. Fig. 8 illustrates the proof that follows. The closed infinite area inside an angle $\angle p_{1} p_{2} p_{3}$, also referred to as a sector, is denoted by $\angle p_{1} p_{2} p_{3}$. In addition, sector $\angle p_{1} p_{2} p_{3} p_{4}$ denotes the intersection of two sectors $\angle p_{1} p_{2} p_{3}$ and $p_{2} p_{3} p_{4}$. First, point $q$ cannot be inside the sector $\angle u^{\prime \prime} y v^{\prime \prime}$. Otherwise, $\|q y\|<\max (\|q u\|,\|q v\|)$ because $y$ is inside the triangle $\triangle q u v$. Thus, $y$ is inside $\operatorname{disk}(u, v, w)$. Then, triangle $\triangle u v w$ should not exist, as $v$ can see $y$ inside $\operatorname{disk}(u, v, w)$. Symmetry implies that also $q$ cannot be inside the sector $\angle u^{\prime \prime \prime} x v^{\prime \prime \prime}$. Second, point $q$ cannot be inside the sector $\angle u v y u^{\prime \prime}$. Otherwise, segment $q c$ intersects $y v$, which implies that $\|v y\|>\|u y\|>1$. It contradicts to $1 \geq\|v y\|$. Similarly, point $q$ also cannot be inside the sector $u^{\prime \prime \prime} x v u^{\prime}$. Third, point $q$ cannot be inside the triangle $\triangle x y v$. Otherwise, $\|q v\| \leq \max (\|v x\|,\|v y\|)<1$. It contradicts to that triangle $\triangle u v w$ is big. Fourth, point $q$ cannot be inside the sector $\angle p^{\prime} x^{\prime} x v^{\prime \prime \prime}$. Indeed, the segment $q v^{\prime}$ cannot intersect the segment $x x^{\prime}$. Otherwise, $\|q x\| \leq\left\|q v^{\prime}\right\| \leq$ $\|q v\|$ and the triangle $\triangle u v w$ should not exist, as $v$ can see $x$ inside $\operatorname{disk}(u, v, w)$. Symmetry also implies that point $q$ cannot be inside the sector $\angle v^{\prime \prime} y y^{\prime} p^{\prime}$. Fifth, $q$ cannot be inside the sector $\angle p^{\prime} y^{\prime} x^{\prime} p^{\prime \prime}$ and the segment $q v^{\prime}$ intersects the segment $x^{\prime} x$. Otherwise, let $q^{\prime}$ be the intersection point of $q v^{\prime}$ and $x^{\prime} x$. Since the biggest edge of $\triangle x^{\prime} y^{\prime} p$ has length 1 , $\|q u\|<1$. Then, from $\left\|q^{\prime} v^{\prime}\right\| \geq\left\|x x^{\prime}\right\|=1$, we have

$$
\begin{aligned}
& \|q u\| \leq\left\|q q^{\prime}\right\|+\left\|q^{\prime} u\right\|<\left\|q q^{\prime}\right\|+1 \leq\left\|q q^{\prime}\right\|+\left\|q^{\prime} v^{\prime}\right\| \\
& =\left\|q v^{\prime}\right\| \leq\|q v\|,
\end{aligned}
$$

contradicting the fact that $\|q u\|=\|q v\|$. Thus, $q$ must be inside the rectangle $x x^{\prime} y^{\prime} y$. Note $\|q x\|>\|q u\| \geq 1$ and $\|q y\|>\|q u\| \geq 1$. Therefore, if $q$ is inside the rectangle $x x^{\prime} y^{\prime} y$, it is inside the triangle $\triangle x^{\prime} y^{\prime} p$. Since the biggest edge of $\triangle x^{\prime} y^{\prime} p$ has length $1,\|q u\|<1$, a contradiction. In conclusion, two big triangles cannot intersect.

Now, we show that the edges of two small triangles cannot intersect, and that the edges of a small triangle cannot intersect a Gabriel edge. Fig. 8 illustrates the proof that follows. As shown above, we can assume that $u$ is inside $\operatorname{disk}(x, y, z)$ and $v$ is outside $\operatorname{disk}(x, y, z)$. Moreover, $\|u x\|>1$ and $\|u y\|>1$. Let $v^{\prime}$ be the intersection of the segment $u v$ with the boundary of $\operatorname{disk}(x, y, z)$. Let $q$ be the center of $\operatorname{disk}(x, y, z)$. Since $\|q y\|=\|q x\|<1$, $u$ cannot be inside the triangle $\triangle q x y$ and, therefore, the segment $u v^{\prime}$ intersects either the segment $x q$ or the segment $y q$. By symmetry, we assume that $u v^{\prime}$ and $q y$ intersect in point $p$. We have that $\left\|q v^{\prime}\right\|=\|q y\|$ and that $\|u y\|>1$. Using triangle inequality for $\triangle q p v^{\prime}$ and $\triangle u p q$, we have $\|q y\|+\left\|u v^{\prime}\right\|>\left\|q v^{\prime}\right\|+\|y u\|$. It deduces that $\left\|u v^{\prime}\right\|>1$ and, therefore $\|u v\|>1$, a contradiction. This completes the proof of the theorem.

\section{ACKNOWLEDGMENTS}

The research of Gruia Calinescu was performed in part while visiting the Department of Combinatorics and Optimization at the University of Waterloo, and partially fupported by NSERC research grants.

\section{RefERENCES}

[1] D.B. Johnson and D.A. Maltz, "Dynamic Source Routing in Ad Hoc Wireless Networks," Mobile Computing, T. Imielinski and H.F. Korth, eds., vol. 353, Kluwer Academic, 1996.

[2] S. Murthy and J. Garcia-Luna-Aceves, "An Efficient Routing Protocol for Wireless Networks," ACM Mobile Networks and Applications J., Special issue on Routing in Mobile Comm. Networks, vol. 1, no. 2, 1996.

[3] V. Park and M. Corson, "A Highly Adaptive Distributed Routing Algorithm for Mobile Wireless Networks," Proc. IEEE Infocom, 1997.

[4] C. Perkins, "Ad-Hoc On-Demand Distance Vector Routing," Proc. MILCOM, Nov. 1997.

[5] C. Perkins and P. Bhagwat, "Highly Dynamic DestinationSequenced Distance-Vector Routing," Proc. ACM SIGCOMM, Oct. 1994.

[6] P. Sinha, R. Sivakumar, and V. Bharghavan, "CEDAR: Core Extraction Distributed Ad Hoc Routing Algorithm," IEEE J. Selected Areas in Comm., vol. 17, no. 8, pp. 1454-1465, Aug. 1999.

[7] E. Royer and C. Toh, "A Review of Current Routing Protocols for Ad-Hoc Mobile Wireless Networks," IEEE Personal Comm., Apr. 1999.

[8] S. Ramanathan and M. Steenstrup, "A Survey of Routing Techniques for Mobile Communication Networks," ACM/Baltzer Mobile Networks and Applications, pp. 89-104, 1996. 
[9] P. Bose and P. Morin, "Online Routing in Triangulations," Proc. 10th Ann. Int'l Symp. Algorithms and Computation, 1999.

[10] I. Stojmenovic and X. Lin, "Loop-Free Hybrid Single-Path/ Flooding Routing Algorithms with Guaranteed Delivery for Wireless Networks," IEEE Trans. Parallel and Distributed Systems, vol. 12, no. 10, 2001.

[11] P. Bose, P. Morin, I. Stojmenovic, and J. Urrutia, "Routing with Guaranteed Delivery in Ad Hoc Wireless Networks," ACM/ Kluwer Wireless Networks, vol. 7, no. 6, pp. 609-616, 2001, Proc. Third Int'l Workshop Discrete Algorithms and Methods for Mobile Computing and Comm., pp. 48-55, 1999.

[12] B. Karp and H.T. Kung, "GPSR: Greedy Perimeter Stateless Routing for Wireless Networks," Proc. ACM/IEEE Int'l Conf. Mobile Computing and Networking, 2000.

[13] L. Barrire, P. Fraigniaud, and L. Narayanan, "Robust PositionBased Routing in Wireless Ad Hoc Networks with Unstable Transmission Ranges," Proc. Fifth Int'l Workshop Discrete Algorithms and Methods for Mobile Computing and Comm., pp. 19-27, 2001.

[14] P. Bose, L. Devroye, W. Evans, and D. Kirkpatrick, "On the Spanning Ratio of Gabriel Graphs and Beta-Skeletons," Proc. Latin Am. Theoretical Informatics Conf., 2002.

[15] D. Eppstein, "Beta-Skeletons Have Unbounded Dilation," Technical Report ICS-TR-96-15, Univ. of California, Irvine, 1996

[16] X.-Y. Li, G. Calinescu, and P.-J. Wan, "Distributed Construction of Planar Spanner and Routing for Ad Hoc Wireless Networks," Proc. 21st IEEE INFOCOM, vol. 3, 2002.

[17] J. Gao, L.J. Guibas, J. Hershburger, L. Zhang, and A. Zhu, "Geometric Spanner for Routing in Mobile Networks," Proc. Second ACM Symp. Mobile Ad Hoc Networking and Computing, 2001.

[18] L. Hu, "Topology Control for Multihop Packet Radio Networks," IEEE Trans. Comm., vol. 41, 1993.

[19] X.-Y. Li, I. Stojmenovic, and Y. Wang, "Partial Delaunay Triangulation and Degree Limited Localized Bluetooth Multihop Scatternet Formation," IEEE Trans. Parallel and Distributed Systems, 2003.

[20] E. Kranakis, H. Singh, and J. Urrutia, "Compass Routing on Geometric Networks," Proc. 11th Canadian Conf. Computational Geometry, pp. 51-54, 1999.

[21] A.C.-C. Yao, "On Constructing Minimum Spanning Trees in kDimensional Spaces and Related Problems," SIAM J. Computing, vol. 11, pp. 721-736, 1982.

[22] J.M. Keil and C.A. Gutwin, "Classes of Graphs which Approximate the Complete Euclidean Graph," Discrete Compuational Geometry, vol. 7, pp. 13-28, 1992.

[23] F.P. Preparata and M.I. Shamos, Computational Geometry: An Introduction. Springer-Verlag, 1985.

[24] D.P. Dobkin, S.J. Friedman, and K.J. Supowit, "Delaunay Graphs are Almost as Good as Complete Graphs," Discrete Computational Geometry, pp. 399-407, 1990.

[25] J.M. Keil and C.A. Gutwin, "The Delaunay Triangulation Closely Approximates the Complete Euclidean Graph," Proc. First Workshop Algorithms Data Structure, 1989.

[26] P.L. Chew, "There is a Planar Graph as Good as the Complete Graph," Proc. Second Symp. Computational Geometry, pp. 169-177, 1986

[27] X.-Y. Li, P.-J. Wan, Y. Wang, and O. Frieder, "Sparse Power Efficient Topology for Wireless Networks," J. Parallel and Distributed Computing, 2002.

[28] K.R. Gabriel and R.R. Sokal, "A New Statistical Approach to Geographic Variation Analysis," Systematic Zoology, vol. 18, pp. 259-278, 1969.

[29] J. Katajainen, "The Region Approach for Computing Relative Neighborhood Graphs in the lp Metric," Computing, vol. 40, pp. 147-161, 1988.

[30] G.T. Toussaint, "The Relative Neighborhood Graph of a Finite Planar Set," Pattern Recognition, vol. 12, no. 4, pp. 261-268, 1980.

[31] W.Z. Wang, X.-Y. Li, K. Moaveninejad, Y. Wang, and W.-Z. Song, "The Spanning Ratios of $\beta$-Skeletons," Proc. Canadian Conf. Computational Geometry, 2003.

[32] T. Lukovszki, "New Results on Geometric Spanners and Their Applications," PhD thesis, Univ. of Paderborn, 1999.

[33] M. Fischer, T. Lukovszki, and M. Ziegler, "Partitioned Neighborhood Spanners of Minimal Outdegree," technical report, Heinz Nixdore Inst., Germany, 1999.

[34] P. Bose, A. Brodnik, S Carlsson, E.D. Demaine, R. Fleischer, A. Lopez-Ortiz, P. Morin, and J.I. Munro, "Online Routing in Convex Subdivisions," Proc. Int'l Symp. Algorithms and Computation, pp. 47$59,2000$.
[35] G. Calinescu, "Computing 2-Hop Neighborhoods in Ad Hoc Wireless Networks." 2002

[36] X.-Y. Li, P.-J. Wan, and O. Frieder, "Coverage in Wireless Ad-Hoc Sensor Networks," IEEE Trans. Computers, vol. 52, no. 6, pp. 753763, 2003

[37] X.-Y. Li, Y. Wang, and O. Frieder, "Efficient Localized Routing for Wireless Ad Hoc Networks," Proc. IEEE Int'l Conf. Comm., 2003.

[38] S. Datta, I. Stojmenovic, and J. Wu, "Internal Node and Shortcut Based Routing with Guaranteed Delivery in Wireless Networks," Proc. IEEE Int'l Conf. Distributed Computing and Systems, pp. 461466, 2001

[39] X.-Y. Li and Y. Wang, "Efficient Construction of Low Weight Bounded Degree Spanner," Proc. Int'l Computing and Combinatorics Conf., 2003.

[40] Y. Wang and X.-Y. Li, "Localized Construction of Bounded Degree and Planar Spanner for Wireless Ad Hoc Networks," submitted for publication, 2003

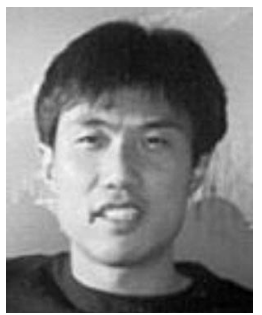

Xiang-Yang Li received the MS (2000) and PhD (2001) degrees in computer science from the University of Illinois at Urbana-Champaign. He received a bachelor degree in computer science and a bachelor degree in business management from Tsinghua University, Peoples Republic of China, in 1995. He has been an assistant professor of computer science at the Illinois Institute of Technology since 2000 . His research interests span the computational geometry, wireless ad hoc networks, optical networks, and cryptography. He is a member of the ACM and IEEE.

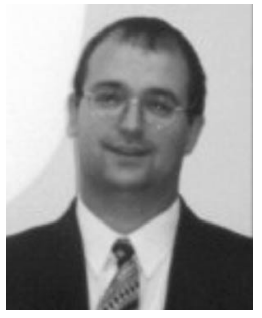

Gruia Calinescu recieved the diploma from the University of Bucharest and the PhD degree from the Georgia Institute of Technology. He is an assistant professor of computer science at the Illinois Institute of Technology. His research interests are in the area of algorithms. He is a member of the ACM and IEEE.

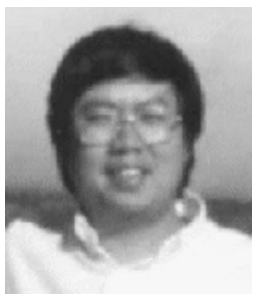

Peng-Jun Wan received the $\mathrm{PhD}$ degree in computer science from University of Minnesota in 1997, the MS degree in operations research and control theory from the Chinese Academy of Science in 1993, and the BS degree in applied mathematics from Qsinghua University in 1990. $\mathrm{He}$ has been an assistant professor in computer science at the Illinois Institute of Technology since 1997. His research interests include optical networks and wireless networks. $\mathrm{He}$ is a member of the IEEE.

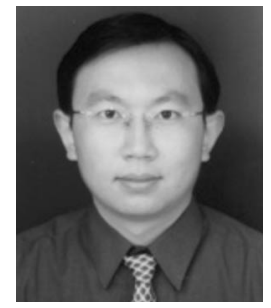

Yu Wang received the BEng degree and the MEng degree from Tsinghua University, Beijing, in 1998 and 2000, both in computer science. He joined the Department of Computer Science Illinois Institute of Technology in 2000 as a PhD student. His current research interests include wireless networks, algorithm design, mobile computing, and artificial intelligence. $\mathrm{He}$ is a member of the ACM, IEEE, and IEEE Communication Society, and a student member of the IEEE Computer Society.

For more information on this or any other computing topic, please visit our Digital Library at http://computer.org/publications/dlib. 